Methods Retrospective data analysis over a 12-month period for in-patients receiving care in a hospital palliative care unit. The primary outcome of this project was to determine the change in frequency of spontaneous bowel movements in the week following the use of naloxegol.

Results Naloxegol was used in 13 people. Nine males (69\%) and 4 females (31\%), mean age 58 (range 47-56). Twelve (93\%) had cancer, 1 (7\%) patient had a diagnosis of COPD. The majority $(n=11,84 \%)$ received two or more laxatives prior to commence naloxegol. Naloxegol was effective in $8(62 \%)$ of people, which was demonstrated by an increase in spontaneous bowel movements. There were no side effects documented for 12 (92\%) individuals. One person $(8 \%)$ developed diarrhoea, which resolved with a dose reduction.

Conclusion Naloxegol was well tolerated and effective in the management of OIC in the majority of this palliative care cohort. This will inform further development of regional guidelines for the management of OIC. Future work is needed to evaluate efficacy and to better understand how naloxegol affects quality of life for people with serious illness.

\section{DEVELOPMENT OF A MORBIDITY AND MORTALITY PROCESS IN A HOSPITAL SPECIALIST PALLIATIVE CARE TEAM}

Kathryn Lockwood, Laura Pal, Jeanna Strutinsky-Mason. University Hospitals of Leicester

\subsection{6/spcare-2020-PCC.155}

Background Morbidity and mortality (M\&M) meetings are a recognised tool for improving quality of care. We have developed an M\&M process within the HSPCT at University Hospitals of Leicester (UHL). Before this the HSPCT contributed to other specialty M\&Ms but did not independently review team involvement in cases using Structured Judgement Review (SJR) methodology.

Aim The aim was to develop our own practice within the team, and to identify areas to improve end of life care across the Trust, by providing feedback into the local Learning from Deaths framework at Trust level.

Methods A quarterly meeting was established with Palliative Medicine Consultant and CNS leads. Referrals were to an electronic mailbox and the leads jointly completed the Structured Judgement Reviews using the Trust template. Cases were presented by the leads who facilitated discussion, concluding with agreed judgements and actions. The finalised SJRs were used as minutes.

Results Over the first three meetings 10 patients were discussed. Referrals increased with 2, 3 and 5 cases discussed in the first, second and third meeting respectively. Referrals were from a variety of sources: 2 from Medical Examiners, 5 by other speciality M\&M leads or consultants and 3 from HSPCT consultants or CNSs. Learning identified was varied and has been grouped into themes: improving outward communication from the HSPCT to specialty teams, areas for changes to current Trust guidelines/policies, areas for education and development.

Conclusions The M\&M has provided a formal way to report back, with case-based evidence, at Trust level and allowed greater communication with other specialities. Doctors and specialist nurses value the time to reflect on clinical aspects of their work. Prioritising protected time for the meeting and enabling clear plans to improve care were key. The meeting has become an established part of the team culture.

\section{A 12-MONTH TRANSFORMATION OF SEVERN HOSPICE DAY UNIT INTO AN ESTABLISHED LIVING WELL DAY SERVICES}

K Maw, R Richardson, D Willis. Severn Hospice

\subsection{6/spcare-2020-PCC.156}

Introduction As people live longer with multidimensional health and end of life care needs, palliative care provision is becoming increasingly complex. Demands on our Services are changing; therefore, as new models of care emerge, enabling patients to live well becomes Severn Hospice's focus.

Method At the beginning of 2018 Day Services were restructured to secure leadership and maintain momentum taking this important initiative forward. Several Hospices across England were scoped, observational studies began and attendance at the APDS over consecutive years maintained in order to network and benchmark progress. Feedback was collated from staff, referrers and patients to establish if services were fit for purpose. Several patient case studies were completed leading to the restructure and rebranding of services.

Results A phased approach towards new structures of care was applied over a 12-month period. This incorporated a triage system with an 8 -week assessment programme, a drop-in networking coffee morning and an education programme. This structure was supplemented by craft workshops, complementary therapy, guest visitors, music workshops, Look Good Feel Better, voluntary agencies, specialist nurses and the Multi-disciplinary team. The overhaul of our literature and promotion was essential in raising the profile of these services and the overall success of this project. The focus remained on patient engagement, enablement and self-care throughout. Staff were supported during the transition with additional training, project days and team away days. All team members tackled complex, unknown territory, receiving outstanding patient feedback in return.

Conclusion From 2019 Day Services continue to successfully coordinate these services and are always looking for ways to increase accessibility and choice for our local population. Referral rates and contacts continue to increase. Joint working with other agencies has improved making the transition between services more.

\section{WHAT IS THE VIRTUE OF VIRTUAL REALITY}

K Maw, R Richardson, D Willis. Severn Hospice

\subsection{6/spcare-2020-PCC.157}

Introduction Our approach to care is to enable those who need our help to live as well as they can within the constraints of a life-limiting illness. How we care for them is personal and ensures we take account of someone's whole wellbeing. The virtual reality project forms a novel yet integral part of our service delivery.

Method The grant from APDS, enabled Severn Hospice to introduce regular VR sessions to patients attending our services. Sessions focused on the needs of the individual within the defined, safe boundaries of Greener Games App - Nature 